\title{
Executive Function in Children and Adolescents with Critical Cyanotic Congenital Heart Disease
}

\author{
Adam R. Cassidy, ${ }^{1}$ Matthew T. White, ${ }^{1}$ David R. DeMaso, ${ }^{1}$ Jane W. Newburger, ${ }^{2}$ And David C. Bellinger ${ }^{1,3}$ \\ ${ }^{1}$ Department of Psychiatry, Boston Children's Hospital, Harvard Medical School, Boston, Massachusetts \\ ${ }^{2}$ Department of Cardiology, Boston Children's Hospital, Harvard Medical School, Boston, Massachusetts \\ ${ }^{3}$ Department of Neurology, Boston Children's Hospital, Harvard Medical School, Boston, Massachusetts
}

(Received June 13, 2014; Final Revision November 3, 2014; Accepted November 3, 2014; First Published Online December 9, 2014)

\begin{abstract}
Children and adolescents with critical cyanotic congenital heart disease (CHD) are at risk for deficits in aspects of executive function (EF). The primary aim of this investigation was to compare EF outcomes in three groups of children/adolescents with severe CHD and controls (ages 10-19 years). Participants included 463 children/adolescents with CHD [dextro-transposition of the great arteries (TGA), $n=139$; tetralogy of Fallot (TOF), $n=68$; and, single-ventricle anatomy requiring Fontan procedure (SVF), $n=145$ ] and 111 controls, who underwent laboratory and informant-based evaluation of EF skills. Rates of EF impairment on D-KEFS measures were nearly twice as high for CHD groups (75-81\%) than controls (43\%). Distinct EF profiles were documented between CHD groups on D-KEFS tasks. Deficits in flexibility/problem-solving and verbally mediated EF skills were documented in all three CHD groups; visuo-spatially mediated EF abilities were impaired in TOF and SVF groups, but preserved in TGA. Parent, teacher, and self-report ratings on the BRIEF highlighted unique patterns of metacognitive and self-regulatory concerns across informants. CHD poses a serious threat to EF development. Greater severity of CHD is associated with worse EF outcomes. With increased understanding of the cognitive and self-regulatory vulnerabilities experienced by children and adolescents with CHD, it may be possible to identify risks early and provide individualized supports to promote optimal neurodevelopment. (JINS, 2015, 20, 34-49)
\end{abstract}

Keywords: Congenital heart defects, Executive function, Transposition of great vessels, Tetralogy of Fallot, Single-ventricle, Fontan procedure

Abbreviations: CHD: congenital heart disease; TGA: dextro-transposition of the great arteries; TOF: tetralogy of Fallot; SVF: single-ventricle cardiac conditions requiring Fontan procedure; HLHS: hypoplastic left heart syndrome; D-KEFS: Delis-Kaplan Executive Function System; BRIEF: Behavior Rating Inventory of Executive Function.

\section{INTRODUCTION}

Congenital heart disease (CHD) includes a diverse array of conditions affecting the structural and/or functional integrity of the heart. Dextro-transposition of the great arteries (TGA), tetralogy of Fallot (TOF), and single-ventricle conditions such as hypoplastic left heart syndrome (HLHS) are among the most serious forms of critical cyanotic CHD (Marino et al., 2012), each requiring early and intensive medical and surgical intervention(s) to sustain life. In TGA, the major blood vessels connecting the systemic and pulmonary blood supplies are transposed. In TOF, four cardiac abnormalities (ventricular septal defect, pulmonary stenosis, right ventricular

Correspondence and reprint requests to: Adam R. Cassidy, Center for Neuropsychology, Department of Psychiatry, Boston Children's Hospital, Boston, MA 02115. E-mail: adam.cassidy@childrens.harvard.edu hypertrophy, and an overriding aorta) undermine heart function and blood flow. In single-ventricle conditions such as HLHS, one ventricle of the heart fails to develop and thus is unable to circulate oxygenated blood to the body; staged palliative surgeries, typically culminating in the Fontan procedure, are often indicated. The incidence of critical cyanotic CHD is approximately 3/1000 live births (Hoffman \& Kaplan, 2002).

Severe CHD poses a serious threat to brain development. The pathophysiological mechanisms underlying neurologic injury in CHD are diverse and not yet fully understood, including not only potential hypoxic/ischemic cascades triggered by hypoperfusion during cardiac surgery but also a wide range of genetic, prenatal, and other pre- and post-operative factors. In at least some forms of severe $\mathrm{CHD}$, atypical brain development is evident prenatally, as early as 25- to 30-weeks gestation (Clouchoux et al., 2013; 
Limperopoulos et al., 2010). Infants with CHD exhibit high rates of microcephaly, hypotonia, and atypical state regulation on clinical examination, and neuroimaging abnormalities such as ischemic infarcts and white matter injury (periventricular leukomalacia) are present in up to $59 \%$ before surgery (Owen, Shevell, Majnemer, \& Limperopoulos, 2011). Relative to controls, the brains of full-term infants with TGA or HLHS are smaller and less mature structurally than those of typically developing infants (Licht et al., 2009), with reduced grey matter volumes particularly in the frontal lobe (Watanabe et al., 2009). Adolescents with corrected TGA (Bellinger et al., 2011) and those with TOF (Bellinger et al., 2014a) exhibit much higher rates of structural MRI abnormalities than controls. Fractional anisotropy on diffusion tensor imaging is significantly reduced in adolescents with TGA, particularly within deep cerebral, cerebellar, and midbrain white matter (Rivkin et al., 2013).

Behavioral studies further evidence the adverse impact of CHD on the developing brain. Within the context of Low Average to Average overall cognitive abilities (Karsdorp, Everaerd, Kindt, \& Mulder, 2007), children/adolescents with $\mathrm{CHD}$, as a group, face increased risk for deficits in speech/ language, sensory/motor, attention, memory, and visualspatial skills (Bellinger et al., 2003, 2009, 2011; Brosig, Mussatto, Kuhn, \& Tweddell, 2007; Calderon et al., 2010; Gaynor et al., 2010; Hovels-Gurich et al., 2002; Miatton et al., 2007a, 2007b; Schaefer et al., 2013). Educational/academic achievement difficulties (Bellinger et al., 2003, 2011), social cognitive deficits (Bellinger, 2008; Bellinger et al., 2011; Calderon et al., 2010), and emotional/behavioral problems (Bellinger et al., 2009; Brosig et al., 2007) are also elevated.

Children and adolescents with CHD are also at risk for deficits in executive function. "Executive function" (EF) refers to a constellation of skills, mediated by densely interconnected neuroanatomical networks involving frontal/ prefrontal (Robbins, 1996), parietal (Champod \& Petrides, 2010), cerebellar (Stoodley \& Schmahmann, 2010), and subcortical structures (Little et al., 2010; Provost, Petrides, \& Monchi, 2010), that are necessary for effective regulation of behavior, emotion, cognition, and social adaptation (Diamond, 2013). Children with severe CHD exhibit problems with inhibitory control (Calderon et al., 2010, 2012; Gaynor et al., 2010, 2014; Miatton et al., 2007a), planning (Bellinger et al., 2003, 2011; Calderon et al., 2010, 2012; Miatton et al., 2007a, 2007b), cognitive flexibility (i.e., switching/shifting; Bellinger et al., 2003, 2011; Calderon et al., 2010, 2012), working memory (Calderon et al., 2010, 2012), and executive attention (Hovels-Gurich et al., 2007). Some also struggle with abstract problem-solving and inferential reasoning, and can have a hard time with efficient retrieval and generation of verbal output (Bellinger et al., 2003). Composite parent and teacher ratings of EF skills confirm self-regulatory and metacognitive difficulties at home and school (Bellinger et al., 2011, 2014a). In contrast, self-report ratings of global EF abilities have generally failed to document significant concerns (Bellinger et al., 2011, 2014a), leading investigators to suggest that "....relying solely on self-reports of patients with congenital heart disease might underestimate the severity of their challenges, at least in the domain of executive functions" (Bellinger et al., 2014a, p. 9).

The primary aim of the current investigation was to compare EF outcomes in four groups of children and adolescents: three with CHD (TGA, TOF, or single-ventricle cardiac conditions culminating in the Fontan procedure) and a group of typically developing controls. Although prior studies have shown that children with severe CHD are at risk for EF deficits, none to date have been designed and/or adequately powered to determine whether distinct forms of CHD are associated with distinct patterns of EF vulnerabilities. In the present study, we operationalized the EF construct broadly, using a combination of well-validated laboratory tasks and parent, teacher, and self-report rating scales, within a large mixedCHD sample of children and adolescents who participated in one of three cardiac neurodevelopmental studies at Boston Children's Hospital.

We hypothesized that children/adolescents with CHD would perform worse on all laboratory EF tasks and would be rated by parents and teachers as having more real-world $\mathrm{EF}$ problems than controls. Self-report ratings were not expected to reflect the same degree of problem severity as parent and teacher reports; nonetheless, by examining perceived concerns across a wide range of specific EF domains, this study provides a more comprehensive test than previous investigations of whether children/adolescents with CHD self-identify EF problems in everyday life. Finally, because data contrasting neurodevelopmental outcomes on the basis of cardiac diagnosis are limited, we conducted exploratory comparisons across CHD groups.

\section{METHOD}

\section{Recruitment and Procedure}

Data were compiled from three large-scale, single-center studies: (1) the Boston Circulatory Arrest Study of children/ adolescents with TGA (Bellinger et al., 2011); (2) a study of children/adolescents with TOF (Bellinger et al., 2014a); and (3) a study of children/adolescents with single-ventricle cardiac anatomy who underwent the Fontan operation (SVF; Bellinger et al., 2014b). All three studies included extensive neuropsychological evaluation (lasting approximately $4 \mathrm{hr}$ ). Psychological measures were administered in a fixed order by a licensed psychologist or supervised research assistant.

Studies were approved by the Institutional Review Board and conducted in accordance with the Helsinki Declaration. Informed consent was obtained from parents of participants; adolescents provided assent.

\section{TGA group}

The Boston Circulatory Arrest Study has been well-described previously (e.g., Bellinger et al., 1995, 2003, 2011; Newburger et al., 1993). Eligible participants included children/adolescents 14-16 years old with TGA who 
underwent the arterial switch operation by 3 months of age. Exclusion criteria included birth weight $<2.5 \mathrm{~kg}$, recognized genetic syndrome, prior heart surgery, or cardiovasculature requiring reconstruction of the aortic arch. Enrolled infants were randomly assigned to receive the arterial switch operation using a strategy of vital organ support of cardiopulmonary bypass with predominant deep hypothermic circulatory arrest or predominant low-flow bypass. Children were followed serially after surgery. Data from the most recent assessment were analyzed in the current study.

\section{TOF group}

Eligible participants included children/adolescents 13-16 years old with TOF (with or without pulmonary atresia) who underwent surgical repair at least 6 months before assessment. Exclusion criteria included diagnosis of trisomy 21 and/or presence of a disorder/device contraindicated for MRI.

\section{SVF group}

Eligible participants included children/adolescents 10-19 years old with single-ventricle cardiac anatomy and who underwent the Fontan procedure, Fontan re-do, or other open-heart surgical procedure at least 6 months before evaluation. Exclusion criteria included history of cardiac transplantation and/or presence of a disorder/device contraindicated for MRI.

\section{Control group}

A total of 111 typically developing children/adolescents 10-19 years old were recruited (61 during the TOF study and 50 during the SVF study) in accordance with admission criteria for the NIH MRI Study of Normal Brain Development (Waber et al., 2007).

\section{Participants}

Among the 497 children/adolescents included in our cohort, 34 (23 TOF and $11 \mathrm{SVF}$ ) had identified genetic/syndromic conditions (e.g., 22q11) and were excluded from analyses. The final pooled sample for the current study included 463 children/adolescents (63.3\% male; 139 TGA, 68 TOF, 145 $\mathrm{SVF}$, and 111 controls) ranging in age from 10 to 19 years $(M=15.17 ; S D=2.04)$. Table 1 presents sample demographic and medical/surgical characteristics.

\section{Measures}

The present investigation used a common subset of laboratory data from the Delis-Kaplan Executive Function System (D-KEFS; Delis, Kaplan, \& Kramer, 2001) and questionnaire data from the Behavior Rating Inventory of Executive Function (BRIEF; Gioia, Isquith, Buy, \& Kenworthy, 2000; Guy, Isquith, \& Geoia, 2004) to examine EF outcomes. Of note, broad neurobehavioral outcomes from the three larger studies, including a D-KEFS composite score and BRIEF General Executive Composite scores, have been described in previous reports (Bellinger et al., 2011, 2014a, 2014b). The present study provides a detailed analysis of EF outcomes, using individual D-KEFS subtest scores and BRIEF subscale/ index scores that have not been published elsewhere.

\section{$D-K E F S$}

The D-KEFS is a widely used battery of laboratory EF tasks. Five subtests were included in the current study. The Verbal Fluency Test is a measure of verbal generativity and switching consisting of 3 conditions (Letter Fluency, Category Fluency, Category Switching). The Design Fluency Test is a measure of visual-spatial generativity and switching consisting of 3 conditions (Filled Dots, Empty Dots, Dot Switching). The Sorting Test is a measure of cognitive flexibility and problemsolving in which participants are asked to sort cards into as many 3-card groups as possible. The Word Context Test is a measure of verbal concept formation and hypothesis-testing requiring participants to determine the definitions of 10 nonsense "mystery" words using a series of context clues. The Tower Test is a measure of visual-spatial planning that requires participants to build a series of towers by arranging flat disks on a board with 3 vertical pegs. Age-referenced scaled scores $(M=10 ; S D=3)$ were included in analyses.

\section{BRIEF}

The BRIEF is a questionnaire designed to solicit information about an individual's use of EF skills in real-world settings. Parent, teacher, and self-report ratings were administered. Age-referenced T-scores $(M=50 ; S D=10)$ were included in analyses. Scores $\geq 65$ are considered "clinically significant;" self-report scores $\geq 60$ may "warrant clinical interpretation" (Guy et al., 2004, p. 16; Gioia et al., 2000).

\section{Data Analysis}

Data analyses were conducted using IBM SPSS Statistics Version 21 and SAS Version 9.3. Variables were examined for normality and outliers; no concerning outliers were present. D-KEFS variables were normally distributed. BRIEF data were significantly positively skewed and could not be normalized adequately with transformation, thereby precluding them from analyses assuming normality. To establish comparability of groups on EF outcomes, six factors potentially related to EF development [socioeconomic status (SES), birth weight, gestational age, age at assessment, sex, and race (white/Caucasian/non-Hispanic vs. nonwhite)] were subjected to separate multivariate analysis of covariance (MANCOVA) models and examined for between-group differences.

Profile analysis was used initially to assess D-KEFS score patterns across CHD (combined) and control groups, and subsequently to compare profiles across CHD subgroups (TGA, TOF, and SVF). This analysis was conducted using a general linear model (PROC GLM in SAS) with the D-KEFS subtests as the outcome variables and with group and other significant covariates included as predictors. Performance on 
Table 1. Sample demographic characteristics by cardiac diagnosis

\begin{tabular}{|c|c|c|c|c|c|}
\hline & $\begin{array}{c}\text { CHD } \\
(n=343-357)\end{array}$ & $\begin{array}{c}\text { Control } \\
(n=101-111)\end{array}$ & $\begin{array}{c}\text { TGA } \\
(n=138-139)\end{array}$ & $\begin{array}{c}\text { TOF } \\
(n=62-68)\end{array}$ & $\begin{array}{c}\text { SVF } \\
(n=141-145)\end{array}$ \\
\hline Family SES ${ }^{\mathrm{a}}$ & $47.88(12.71)$ & $53.06(9.55)$ & $45.81(12.18)$ & $48.65(11.95)$ & $49.50(13.34)$ \\
\hline Gestational age (weeks) & $39.34(1.98)$ & $39.58(1.30)$ & $39.75(1.25)$ & $39.17(2.49)$ & $39.00(2.24)$ \\
\hline Birth weight $(k g)$ & $3.39(0.58)$ & $3.48(0.59)$ & $3.55(0.45)$ & $3.21(0.67)$ & $3.31(0.62)$ \\
\hline Sex: male $n(\%)$ & $234(66.5)$ & $59(53.2)$ & $106(76.3)$ & $38(55.9)$ & $90(62.1)$ \\
\hline \multicolumn{6}{|l|}{ Race/Ethnicity $n(\%)$} \\
\hline White/Caucasian/Non-Hispanic & $303(86.1)$ & $89(80.2)$ & $126(90.6)$ & $59(86.8)$ & $118(81.4)$ \\
\hline Nonwhite: & 49 (13.9) & $22(19.8)$ & $13(9.4)$ & $9(13.2)$ & $27(18.6)$ \\
\hline White/Hispanic & $26(7.4)$ & $3(2.7)$ & $5(3.6)$ & $5(7.4)$ & $16(11.0)$ \\
\hline Asian & $6(1.7)$ & $5(4.5)$ & $2(1.4)$ & $0(0.0)$ & $4(2.8)$ \\
\hline Black & $9(2.6)$ & $9(8.1)$ & $2(1.4)$ & $2(2.9)$ & $5(3.4)$ \\
\hline Pacific Islander & $1(0.3)$ & $0(0.0)$ & $1(0.7)$ & $0(0.0)$ & $0(0.0)$ \\
\hline Biracial/Mixed Race & $7(2.0)$ & $5(4.5)$ & $3(2.2)$ & $2(2.9)$ & $2(1.4)$ \\
\hline Age at assessment (years) & $15.13(2.11)$ & $15.30(1.81)$ & $16.08(0.51)$ & $14.67(1.18)$ & $14.44(2.91)$ \\
\hline One-minute Apgar score ${ }^{\mathrm{b}}$ & $7.66(1.37)$ & $8.97(1.35)$ & $7.50(1.35)$ & $7.68(1.73)$ & $7.86(1.30)$ \\
\hline Five-minute Apgar score ${ }^{c}$ & $8.50(0.84)$ & $9.45(0.51)$ & $8.32(0.90)$ & $8.83(0.65)$ & $8.65(0.75)$ \\
\hline Age at first operation (days) & $62.17(151.12)$ & - & $9.89(11.76)$ & $185.56(233.41)$ & $54.42(142.47)$ \\
\hline Total cardiac operations $M d n(\min -\max )$ & $2(1-7)$ & - & $1(1-4)$ & $2(1-7)$ & $3(1-5)$ \\
\hline
\end{tabular}

Note. $\mathrm{CHD}=$ congenital heart disease; TGA $=$ dextro-transposition of the great arteries; $\mathrm{TOF}=$ tetralogy of Fallot; $\mathrm{SVF}=$ single-ventricle children/adolescents who underwent the Fontan procedure; SES = family socioeconomic status according to Hollingshead Four Factor Index of Social Status (1975). The CHD category includes all participants in the TGA, TOF, and SVF groups. Some demographic data were missing; therefore, sample sizes, which are provided above as min-max, depict valid $n$ s by group. Unless otherwise specified, results are presented as mean (standard deviation).

${ }^{\mathrm{a}}$ Hollingshead, A. A. (1975). Four-factor index of social status. Unpublished manuscript, Yale University, New Haven, CT.

${ }^{\mathrm{b}} n \mathrm{~s}: \mathrm{CHD}=256 ;$ Control $=30 ; \mathrm{TGA}=127 ; \mathrm{TOF}=25 ; \mathrm{SVF}=104$.

${ }^{c} n \mathrm{~s}: \mathrm{CHD}=252 ;$ Control $=29 ; \mathrm{TGA}=126 ; \mathrm{TOF}=23 ; \mathrm{SVF}=103$

D-KEFS subtests was compared across groups using contrasts from the profile analysis with a Bonferroni correction for pairwise comparisons. D-KEFS subtest scores were then dichotomized using a cutoff of $1.5 S D$ below population mean (scaled scores $\leq 6$ ) to denote impairment. BRIEF data were also dichotomized using accepted cutoff scores. Logistic regression, controlling for significant covariates, was used to compare the odds of scoring within the impaired/elevated range between $\mathrm{CHD}$ groups and controls on D-KEFS/BRIEF measures. Paired t-tests (calculated separately for $\mathrm{CHD}$ and control groups) were performed to compare self-report versus parent/teacher BRIEF ratings. We used Benjamini and Hochberg's (1995) false discovery rate procedure to limit the chance of reporting a falsely significant result to be no more than $5 \%$. We first determined significance of the overall group effect for each model and then, if significant, conducted pairwise comparisons between groups to identify significant group differences. Using this procedure, a $p$-value $\leq .031$ was considered statistically significant. In an exploratory analysis, Spearman partial correlation coefficients were calculated to evaluate associations between D-KEFS and BRIEF variables.

\section{RESULTS}

\section{Comparability of Groups}

SES, birth weight, gestational age, age at assessment, sex, and race were examined as potential covariates in four separate MANCOVA models: (1) all D-KEFS variables, (2) BRIEF
Parent, (3) BRIEF Teacher, and (4) BRIEF Self-Report. Groups did not differ in gestational age or birth weight for any EF outcome. Significant findings were as follows: for D-KEFS: SES $[F(11,413)=4.91 ; p<.001]$, age at assessment $[F(11,415)=$ $1.81 ; p=.05]$, sex $[F(11,415)=2.84 ; p=.001]$, and race $[F(11,415)=2.70 ; \quad p=.002] ;$ for BRIEF-Parent: SES $[F(8,439)=5.91 ; p<.001]$ and $\operatorname{sex}[F(8,441)=2.66$; $p=.007] ;$ for BRIEF-Teacher: $\operatorname{sex}[F(8,235)=2.86$; $p=.005]$ and race $[F(8,235)=2.46 ; p=.01]$; for BRIEFSelf: SES $[F(8,416)=2.81 ; p=.005]$. Significant factors for each respective EF outcome source were included as covariates in primary analyses. Consistent with the rationale proposed by Dennis et al. (2009), IQ was not included as a covariate in any analysis (see also Miller, Loya, \& Hinshaw, 2013).

\section{Primary Analyses}

\section{D-KEFS task performance}

Most D-KEFS subtest scores were within the average range, except for the Sorting Recognition score, which was below average in some CHD groups (Table 2). Performance profiles are depicted graphically in Figure 1. Profile analysis indicated that D-KEFS score patterns between combined CHD and control participants were not parallel $[F(11,410)=2.65$; $p<.001]$. Looking specifically at score patterns across the TGA, TOF, and SVF groups, likewise revealed a lack of parallelism, $F(22,820)=3.16 ; p<.001$; the TGA profile differed significantly from the $\operatorname{SVF}[F(11,410)=4.53$; 
Table 2. D-KEFS subtest performance and impaired subtest score percentages by cardiac diagnosis

\begin{tabular}{|c|c|c|c|c|c|c|c|c|}
\hline \multirow[b]{2}{*}{ D-KEFS Subtest } & \multirow{2}{*}{$\frac{\text { CHD }}{n=330-351}$} & \multirow{2}{*}{$\frac{\text { Control }}{n=102-111}$} & \multirow{2}{*}{$\frac{\text { TGA }}{n=128-139}$} & \multirow{2}{*}{$\frac{\text { TOF }}{n=62-68}$} & \multirow{2}{*}{$\frac{\mathrm{SVF}}{n=140-144}$} & \multicolumn{2}{|c|}{ CHD vs. control } & \multirow[b]{2}{*}{ Pairwise comparisons (Cohen's $d$ ) } \\
\hline & & & & & & $p$-value & Cohen's $d$ & \\
\hline \multicolumn{9}{|l|}{ VERBAL } \\
\hline \multicolumn{9}{|l|}{ Verbal Fluency Test } \\
\hline Letter Fluency & $9.25(3.49)$ & $11.77(3.40)$ & $8.63(3.51)$ & $9.49(3.56)$ & $9.73(3.37)$ & $<.001$ & .60 & $\mathrm{C}>\mathrm{TGA}^{* * *}(.79), \mathrm{SVF}^{* * *}(.49)$ \\
\hline Category Fluency & $9.94(3.31)$ & $11.73(3.34)$ & $9.80(3.14)$ & $9.96(3.18)$ & $10.08(3.53)$ & .001 & .41 & $\mathrm{C}>\mathrm{TGA}^{* *}(.45), \mathrm{SVF}^{*}(.40)$ \\
\hline Cat. Switch Correct & $8.87(3.35)$ & $11.20(3.42)$ & $8.57(3.26)$ & $8.87(3.48)$ & $9.17(3.37)$ & $<.001$ & .64 & $\mathrm{C}>\mathrm{TGA}^{* * *}(.69), \mathrm{TOF}^{* *}(.59), \mathrm{SVF}^{* * *}(.59)$ \\
\hline \multicolumn{9}{|l|}{ Word Context Test } \\
\hline Total Consec. Correct & $8.42(3.54)$ & $11.09(2.42)$ & $8.66(3.24)$ & $8.82(3.91)$ & $8.01(3.61)$ & $<.001$ & .78 & $\mathrm{C}>\mathrm{TGA}^{* * *}(.72), \mathrm{TOF}^{* *}(.55), \mathrm{SVF}^{* * *}(.91)$ \\
\hline \multicolumn{9}{|l|}{ VISUO-SPATIAL } \\
\hline \multicolumn{9}{|l|}{ Design Fluency Test } \\
\hline Filled Dots & $9.45(2.99)$ & $10.67(2.81)$ & $10.29(2.94)$ & $8.87(2.99)$ & $8.92(2.86)$ & .002 & .35 & $\mathrm{C}>\mathrm{TOF}^{*}(.47), \mathrm{SVF}^{* * *}(.59) ; \mathbf{T G A}>\mathrm{TOF}^{*}(.45), \mathrm{SVF}^{* * *}(.57)$ \\
\hline Empty Dots & $9.37(2.96)$ & $11.05(2.90)$ & $9.96(3.10)$ & $8.91(3.07)$ & $9.02(2.69)$ & $<.001$ & .53 & $\mathrm{C}>\mathrm{TOF}^{* * *}(.64), \mathrm{SVF}^{* * *}(.71) ; \mathbf{T G A}>\mathbf{S V F}^{* *}(. \mathbf{4 5})$ \\
\hline Dot Switching & $9.65(3.16)$ & $11.52(2.88)$ & $10.38(2.97)$ & $9.46(3.71)$ & $9.03(2.92)$ & $<.001$ & .58 & $\mathrm{C}>\mathrm{TOF}^{* *}(.55), \mathrm{SVF}^{* * *}(.82) ; \mathbf{T G A}>\mathbf{S V F}^{* * *}(. \mathbf{5 0})$ \\
\hline \multicolumn{9}{|l|}{ Tower Test } \\
\hline Total Ach. Score & $9.20(2.74)$ & $10.32(2.20)$ & $9.68(2.46)$ & $8.91(3.31)$ & $8.87(2.65)$ & $<.001$ & .45 & $\mathrm{C}>\mathrm{SVF}^{* * *}(.59)$ \\
\hline TPMR & $9.45(2.55)$ & $10.71(1.29)$ & $9.48(2.31)$ & $9.39(2.73)$ & $9.45(2.69)$ & $<.001$ & .55 & $\mathrm{C}>\mathrm{TGA}^{* * *}(.54) ; \mathrm{TOF}^{* *}(.54), \mathrm{SVF}^{* * *}(.54)$ \\
\hline MAR & $9.57(2.85)$ & $9.19(2.62)$ & $9.93(2.50)$ & $9.52(3.44)$ & $9.26(2.87)$ & .112 & .18 & - \\
\hline \multicolumn{9}{|l|}{ COMBINED } \\
\hline \multicolumn{9}{|l|}{ Sorting Test } \\
\hline Conf. Correct Sorts & $8.10(3.03)$ & $9.95(2.37)$ & $7.88(2.63)$ & $7.81(3.56)$ & $8.44(3.10)$ & $<.001$ & .64 & $\mathrm{C}>\mathrm{TGA}^{* * *}(.60), \mathrm{TOF}^{* * *}(.73), \mathrm{SVF}^{* * *}(.61)$ \\
\hline Sort Recognition & $6.75(3.33)$ & $9.46(2.82)$ & $6.76(3.32)$ & $7.37(3.84)$ & $6.47(3.07)$ & $<.001$ & .81 & $\mathrm{C}>\mathrm{TGA}^{* * *}(.78), \mathrm{TOF}^{* *}(.55), \mathrm{SVF}^{* * *}(.94)$ \\
\hline $\begin{array}{l}\% \text { scoring } \geq 1.5 S D \text { s below } \\
\text { the population } M \\
\text { on at least } 1 \text { subtest }\end{array}$ & 77.5 & 43.6 & 80.5 & 77.0 & 75.0 & & & \\
\hline
\end{tabular}

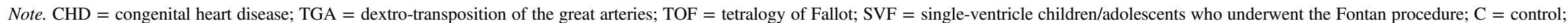

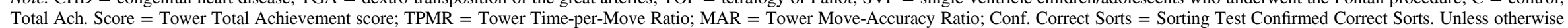

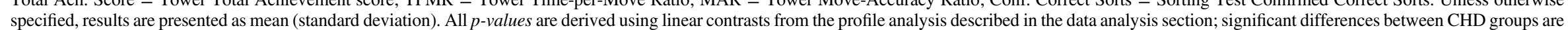

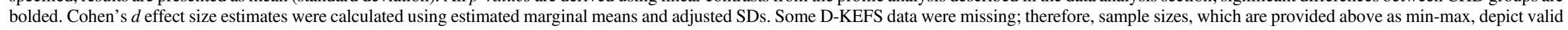
$n$ s by group.

${ }^{*} p \leq .031$.

$* * p \leq .01$.

$* * * p \leq .001$. 


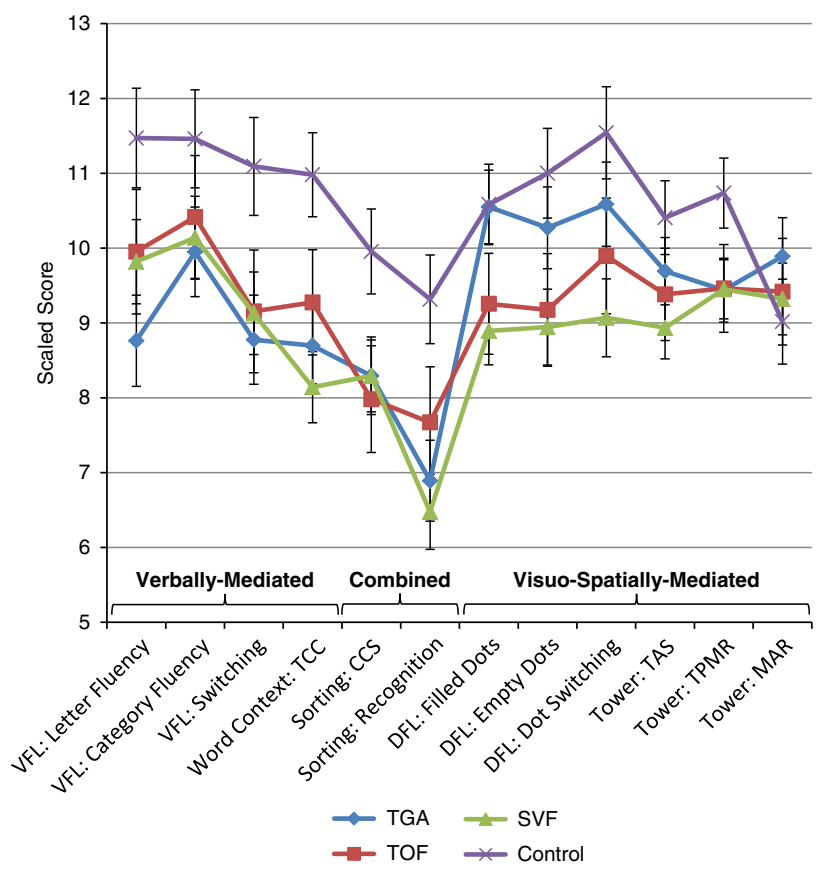

Fig. 1. Estimated marginal means of D-KEFS tasks in cardiac and control groups, controlling for SES, age, sex, and race (error bars represent $95 \%$ confidence intervals). TGA = dextro-transposition of the great arteries; TOF = tetralogy of Fallot; SVF = singleventricle children/adolescents who underwent the Fontan procedure; VFL $=$ Verbal Fluency; $\mathrm{TCC}=$ Total Consecutively Correct; $\quad \mathrm{CCS}=$ Confirmed $\quad$ Correct Sorts; $\mathrm{DFL}=$ Design Fluency; TAS $=$ Total Achievement Score; TPMR $=$ Time per Move Ratio; MAR = Move-Accuracy Ratio.

$p<.001]$ and TOF $[F(11,410)=2.65 ; p=.003]$ profiles, and the TOF and SVF profiles were also significantly different $[F(11,410)=2.01 ; p=.026]$.

Linear contrasts comparing TGA, TOF, SVF, and controls identified significant group differences. At least one CHD group performed significantly worse than controls for all D-KEFS measures except the Tower MAR, which was not statistically different among the groups. See Table 2 for a summary of significant pairwise differences between groups. No significant differences between CHD groups were noted on verbally mediated EF tasks (Verbal Fluency and Word Context Tests) or on tasks with combined verbal/visuo-spatial demands (Sorting Test). In contrast, TGA- (and, in some instances, TOF-) group performance was relatively secure on most visuospatial EF tasks. On the Design Fluency Test, TGA-group performance was not statistically different than controls-and better than the SVF group-across all three trials; the TGA group also outperformed the TOF group on Filled Dots trial. On the Tower Test, TGA- and TOF-group Total Achievement Scores were not statistically different than controls; however, all three CHD groups scored lower than controls on Time-perMove Ratio, indicating greater efficiency in task-completion among healthy children/adolescents than those with CHD. Pairwise CHD group differences were moderate in effect size.

Table 3 presents results from the logistic regression models used to compare the odds of impaired D-KEFS task performance among the control and CHD groups. Looking first at verbal EF tasks, the odds of impairment on the Category Fluency task were no worse among CHD groups than controls. In contrast, TGA and TOF groups had greater odds of impaired Letter Fluency than controls; odds of impaired Letter Fluency in the SVF group were lower than in the TGA group. All three CHD groups had greater odds of impaired Category Switching compared to controls, arguably the most demanding of the verbal fluency tasks. The highest odds of impairment were on the Word Context Test, with the SVF group significantly higher than both control and TGA participants.

On visuo-spatial EF tasks, the odds of impairment on all three Design Fluency trials and two out of three Tower measures were statistically greater among TOF and SVF groups than controls; the TGA group was more likely than controls to score within the impaired range on Empty Dots, but was otherwise at no greater risk for impaired Design Fluency or Tower Test performance than controls. Moreover, the TGA group was at lower risk than TOF for impairment on the Dot Switching trial, and at lower risk than both TOF and SVF groups for impairment on Filled Dots trial.

Finally, the odds of impairment on the Sorting Test were higher in all three CHD groups than controls.

BRIEF reports. Group-level means were within normal limits (Table 4). Percentages of children/adolescents obtaining at least one elevated subscale differed markedly across CHD and control groups for parent and teacher ratings. Self-report ratings with at least one elevated score also differed from controls using a clinical cutoff score of $\geq 65$, but more closely approximated parent/teacher percentages using a relaxed cutoff score of $\geq 60$.

Results of logistic regression analyses and pairwise comparisons are presented in Table 5. On parent report, the odds of being rated as having clinically significant metacognitive and self-regulatory problems were statistically higher among CHD groups than controls for most domains. Inhibition was the only exception; odds of parent-rated inhibitory control problems were greater than controls for the SVF group, but no different for TGA and TOF groups. Children/adolescents in the SVF group were also statistically more likely than those in the TGA group to have parentreported problems with initiation and working memory.

On teacher report, the odds of being rated as having clinically significant metacognitive problems were statistically higher among CHD groups than controls for most domains. The TOF group had significantly greater odds of having problems with inhibition than both control and TGA groups. Otherwise, the odds of clinically significant self-regulatory problems were not statistically greater among children/adolescents with CHD than controls, according to teachers.

Logistic regression analyses of self-report ratings revealed no significant differences in odds of obtaining ratings $\geq 65$ across groups. Using a cutoff score of $\geq 60$, however, the odds of self-identifying problems with shifting and emotion regulation were statistically greater among all three CHD groups than controls. TGA participants were more likely than controls 
Table 3. Odds of impairment on D-KEFS subtest by CHD group as compared to controls

\begin{tabular}{|c|c|c|c|c|c|c|c|c|c|c|}
\hline \multirow[b]{2}{*}{ D-KEFS Subtest } & \multicolumn{3}{|c|}{ TGA } & \multicolumn{3}{|c|}{ TOF } & \multicolumn{3}{|c|}{ SVF } & \multirow[b]{2}{*}{ CHD-group pairwise comparisons } \\
\hline & $p$ & OR & $95 \% \mathrm{CI}$ & $p$ & OR & $95 \% \mathrm{CI}$ & $p$ & OR & $95 \% \mathrm{CI}$ & \\
\hline \multicolumn{11}{|l|}{ VERBAL } \\
\hline \multicolumn{11}{|l|}{ Verbal Fluency Test } \\
\hline Letter Fluency & $<.001$ & 5.36 & $2.27-12.66$ & .004 & 4.02 & $1.54-10.49$ & .07 & 2.33 & $0.95-5.72$ & $\mathrm{TGA}>\mathrm{SVF}^{* *}(\mathrm{OR}=1.15)$ \\
\hline Category Fluency & .11 & 2.21 & $0.84-5.86$ & .13 & 2.33 & $0.78-6.95$ & .04 & 2.76 & $1.07-7.14$ & - \\
\hline Cat. Switch Correct & .002 & 3.29 & $1.57-6.89$ & .02 & 2.74 & $1.18-6.37$ & .01 & 2.62 & $1.25-5.48$ & - \\
\hline \multicolumn{11}{|l|}{ Word Context Test } \\
\hline TCC & .001 & 6.59 & $2.13-20.37$ & $<.001$ & 12.37 & $3.81-40.19$ & $<.001$ & 16.15 & $5.40-48.31$ & $\mathrm{SVF}>\mathrm{TGA} * * *(\mathrm{OR}=1.18)$ \\
\hline \multicolumn{11}{|l|}{ VISUO-SPATIAL } \\
\hline \multicolumn{11}{|l|}{ Design Fluency Test } \\
\hline Filled Dots & .35 & 1.76 & $0.54-5.73$ & .003 & 5.90 & $1.81-19.26$ & .001 & 6.72 & $2.26-19.99$ & $\begin{array}{l}\mathrm{TOF}>\mathrm{TGA}^{*}(\mathrm{OR}=1.13) \\
\quad \mathrm{SVF}>\mathrm{TGA}^{* * *}(\mathrm{OR}=1.16)\end{array}$ \\
\hline Empty Dots & .004 & 6.50 & $1.79-23.60$ & .001 & 10.04 & $2.69-37.44$ & $<.001$ & 9.07 & $2.63-31.29$ & - \\
\hline Dot Switching & .26 & 1.79 & $0.66-4.87$ & .002 & 4.91 & $1.80-13.37$ & .006 & 3.64 & $1.44-9.22$ & $\mathrm{TOF}>\mathrm{TGA}^{*}(\mathrm{OR}=1.14)$ \\
\hline \multicolumn{11}{|l|}{ Tower Test } \\
\hline Total Achievement & .19 & 2.26 & $0.68-7.55$ & .008 & 5.24 & $1.55-17.72$ & .005 & 4.90 & $1.61-14.94$ & - \\
\hline TPMR & .05 & 4.72 & $0.98-22.74$ & .02 & 6.66 & $1.33-33.36$ & .02 & 6.13 & $1.35-27.79$ & - \\
\hline MAR & .07 & 0.48 & $0.22-1.05$ & .92 & 1.05 & $0.46-2.39$ & .65 & 0.85 & $0.43-1.69$ & - \\
\hline \multicolumn{11}{|l|}{ COMBINED } \\
\hline \multicolumn{11}{|l|}{ Sorting Test } \\
\hline $\mathrm{CCS}$ & $<.001$ & 5.15 & $2.12-12.53$ & $<.001$ & 7.58 & $2.93-19.57$ & .009 & 3.30 & $1.35-8.05$ & \multirow{2}{*}{$\begin{array}{c}\mathrm{TOF}>\mathrm{SVF} *(\mathrm{OR}=1.16) \\
-\end{array}$} \\
\hline Sort Recognition & $<.001$ & 4.80 & $2.49-9.26$ & $<.001$ & 4.09 & $1.94-8.62$ & $<.001$ & 4.66 & $2.45-8.88$ & \\
\hline
\end{tabular}

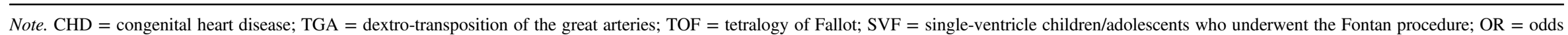

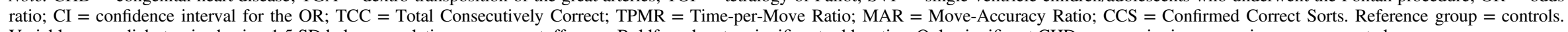
Variables were dichotomized using 1.5 SD below population mean as cutoff score. Boldface denotes significant odds ratios. Only significant CHD-group pairwise comparisons are presented.

$* p \leq .031$.

$* * p \leq .01$.

$* * * p \leq .001$. 
Table 4. BRIEF descriptive results and elevated subscale score percentages by CHD diagnosis

\begin{tabular}{|c|c|c|c|c|c|}
\hline & CHD & Control & TGA & TOF & SVF \\
\hline BRIEF Parent-Report & $(n=348)$ & $(n=105)$ & $(n=138)$ & $(n=67)$ & $(n=143)$ \\
\hline Behavior Regulation Index & $52.88(12.31)$ & $44.47(7.21)$ & $51.50(11.76)$ & $52.63(13.23)$ & $54.32(12.31)$ \\
\hline Inhibit & $52.07(12.04)$ & $46.22(7.62)$ & $50.85(10.80)$ & $52.18(13.08)$ & $53.20(12.62)$ \\
\hline Shift & $53.74(12.33)$ & $44.96(8.09)$ & $52.41(11.96)$ & $53.55(13.14)$ & $55.11(12.24)$ \\
\hline Emotional Control & $52.03(12.04)$ & $44.55(6.76)$ & $51.01(11.19)$ & $51.70(12.69)$ & $53.18(12.51)$ \\
\hline Metacognition Index & $57.21(12.17)$ & $45.73(8.74)$ & $56.02(12.04)$ & $57.07(12.80)$ & $58.42(11.94)$ \\
\hline Initiate & $56.19(12.33)$ & $45.87(9.32)$ & $54.59(12.42)$ & $55.79(12.50)$ & $57.92(12.02)$ \\
\hline Working Memory & $57.15(13.66)$ & $45.37(8.74)$ & $55.92(14.11)$ & $56.64(14.16)$ & $58.57(12.94)$ \\
\hline Planning/Organization & $56.68(12.36)$ & $45.56(8.14)$ & $55.39(12.05)$ & $56.09(12.91)$ & $58.20(12.31)$ \\
\hline Organization of Materials & $54.52(10.55)$ & $48.97(9.73)$ & $54.21(10.16)$ & $55.57(10.49)$ & $54.32(10.98)$ \\
\hline Monitor & $56.20(12.13)$ & $45.57(9.00)$ & $56.06(11.51)$ & $56.01(13.13)$ & $56.43(12.31)$ \\
\hline$\%$ rated $\geq 1.5 S D$ s above the population mean on at least 1 subscale & 56.3 & 15.2 & 50.7 & 56.7 & 61.5 \\
\hline BRIEF Teacher-Report & $(n=205-207)$ & $(n=42)$ & $(n=78-79)$ & $(n=44-45)$ & $(n=83)$ \\
\hline Behavior Regulation Index & $56.77(15.58)$ & $51.38(9.31)$ & $55.21(13.32)$ & $59.93(19.93)$ & $56.55(14.88)$ \\
\hline Inhibit & $54.55(13.93)$ & $50.52(7.66)$ & $52.11(10.38)$ & $57.16(17.01)$ & $55.45(14.81)$ \\
\hline Shift & $59.72(19.50)$ & $51.90(13.40)$ & $60.22(20.58)$ & $62.00(22.30)$ & $58.05(16.77)$ \\
\hline Emotional Control & $54.75(15.33)$ & $51.07(10.37)$ & $52.96(13.17)$ & $57.89(19.39)$ & $54.75(14.66)$ \\
\hline Metacognition Index & $61.42(16.71)$ & $52.33(10.67)$ & $60.53(16.40)$ & $61.76(18.43)$ & $62.07(16.17)$ \\
\hline Initiate & $60.03(15.88)$ & $51.02(10.21)$ & $59.97(16.62)$ & $58.67(15.73)$ & $60.83(15.38)$ \\
\hline Working Memory & $61.90(17.62)$ & $52.95(11.09)$ & $60.57(16.76)$ & $62.47(19.88)$ & $62.86(17.27)$ \\
\hline Planning/Organization & $60.14(15.45)$ & $51.83(11.35)$ & $59.78(16.03)$ & $61.22(17.05)$ & $59.89(14.09)$ \\
\hline Organization of Materials & $59.21(19.42)$ & $51.74(11.59)$ & $57.89(18.06)$ & $58.87(20.78)$ & $60.66(20.05)$ \\
\hline Monitor & $59.36(15.28)$ & $52.57(9.97)$ & $57.71(13.62)$ & $60.56(17.56)$ & $60.29(15.52)$ \\
\hline$\%$ rated $\geq 1.5 S D$ s above the population mean on at least 1 subscale & 57.1 & 28.6 & 52.6 & 54.5 & 62.7 \\
\hline BRIEF Self-Report & $(n=321)$ & $(n=109)$ & $(n=136)$ & $(n=65)$ & $(n=120)$ \\
\hline Behavior Regulation Index & $49.32(11.14)$ & $43.65(9.28)$ & $49.79(11.61)$ & $49.23(11.17)$ & $48.83(10.64)$ \\
\hline Inhibit & $49.10(10.99)$ & $45.23(8.63)$ & $49.90(11.48)$ & $49.20(11.71)$ & $48.13(9.98)$ \\
\hline Shift & $49.81(11.22)$ & $43.86(10.10)$ & $49.55(11.36)$ & $49.43(10.88)$ & $50.32(11.32)$ \\
\hline Emotional Control & $49.14(10.50)$ & $44.62(8.62)$ & $49.24(10.84)$ & $49.25(10.71)$ & $48.98(10.08)$ \\
\hline Monitor & $50.04(10.39)$ & $45.50(9.55)$ & $50.68(11.27)$ & $50.09(10.60)$ & $49.29(9.19)$ \\
\hline Metacognition Index & $50.86(11.33)$ & $46.18(10.35)$ & $51.40(11.55)$ & $50.37(10.42)$ & $50.51(11.62)$ \\
\hline Working Memory & $51.38(11.44)$ & $47.25(11.44)$ & $51.56(11.38)$ & $51.12(12.20)$ & $51.32(11.18)$ \\
\hline Planning/Organization & $49.99(10.75)$ & $45.45(9.31)$ & $50.95(11.34)$ & $49.08(9.51)$ & $49.39(10.71)$ \\
\hline Organization of Materials & $49.78(10.03)$ & $48.43(10.01)$ & $50.80(10.05)$ & $50.02(9.74)$ & $48.49(10.09)$ \\
\hline Task Completion & $51.69(11.52)$ & $46.33(10.09)$ & $51.59(11.52)$ & $51.12(11.17)$ & $52.13(11.79)$ \\
\hline$\%$ rated $\geq 1.5(1.0) S D$ s above the population mean on at least 1 subscale & $30.8(49.5)$ & $15.6(22.9)$ & $35.3(52.9)$ & $27.7(49.2)$ & $27.5(45.8)$ \\
\hline
\end{tabular}

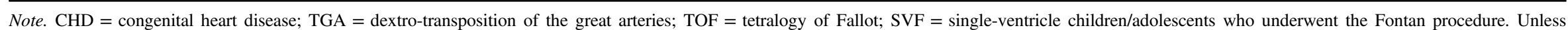
otherwise specified, results are presented as mean (standard deviation). Some BRIEF Teacher-report data were missing; therefore, sample sizes, which are provided above as min-max, depict valid $n$ s by group. 
Table 5. Odds of clinically significant problems on BRIEF subscale by CHD group as compared to controls

\begin{tabular}{|c|c|c|c|c|c|c|c|c|c|c|}
\hline & \multicolumn{3}{|c|}{ TGA } & \multicolumn{3}{|c|}{ TOF } & \multicolumn{3}{|c|}{ SVF } & \multirow[b]{2}{*}{ CHD-group pairwise comparisons } \\
\hline & $p$ & OR & $95 \% \mathrm{CI}$ & $p$ & OR & $95 \% \mathrm{CI}$ & $p$ & OR & $95 \% \mathrm{CI}$ & \\
\hline \multicolumn{11}{|l|}{ BRIEF Parent } \\
\hline Inhibit & .30 & 1.77 & $0.61-5.14$ & .04 & 3.21 & $1.05-9.85$ & .003 & 4.46 & $1.65-12.07$ & - \\
\hline Shift & .008 & 5.43 & $1.56-18.87$ & $<.001$ & 9.81 & $2.72-35.41$ & .001 & 8.28 & $2.44-28.04$ & - \\
\hline Emot. Control & .02 & 6.01 & $1.35-26.82$ & .02 & 6.51 & $1.35-31.50$ & .003 & 9.57 & $2.20-41.67$ & - \\
\hline Initiate & .01 & 3.14 & $1.30-7.60$ & .002 & 4.61 & $1.79-11.86$ & $<.001$ & 6.87 & $2.95-15.99$ & $\mathrm{SVF}>\mathrm{TGA}^{* *}(\mathrm{OR}=1.16)$ \\
\hline Working Memory & .001 & 8.33 & $2.45-28.32$ & $<.001$ & 13.40 & $3.78-47.51$ & $<.001$ & 17.80 & $5.35-59.22$ & $\mathrm{SVF}>\mathrm{TGA}^{* *}(\mathrm{OR}=1.16)$ \\
\hline Plan/Organize & $<.001$ & 8.03 & $2.74-23.56$ & $<.001$ & 10.30 & $3.33-31.87$ & $<.001$ & 13.13 & $4.55-37.87$ & - \\
\hline Org. of Materials & .005 & 3.09 & $1.40-6.82$ & .01 & 3.08 & $1.26-7.51$ & .005 & 3.08 & $1.40-6.76$ & - \\
\hline Monitor & $<.001$ & 6.27 & $2.54-15.50$ & $<.001$ & 7.02 & $2.65-18.64$ & $<.001$ & 7.10 & $2.89-17.42$ & - \\
\hline \multicolumn{11}{|l|}{ BRIEF Teacher } \\
\hline Inhibit & .84 & 1.17 & $0.27-5.00$ & .01 & 5.81 & $1.49-22.68$ & .13 & 2.80 & $0.75-10.43$ & $\mathrm{TOF}>\mathrm{TGA}^{* *}(\mathrm{OR}=1.23)$ \\
\hline Shift & .07 & 2.53 & $0.92-6.91$ & .08 & 2.64 & $0.89-7.85$ & .07 & 2.54 & $0.94-6.86$ & - \\
\hline Emot. Control & .07 & 3.31 & $0.89-12.34$ & .05 & 3.95 & $0.99-15.72$ & .12 & 2.80 & $0.76-10.38$ & - \\
\hline Initiate & .01 & 3.52 & $1.31-9.47$ & .06 & 2.83 & $0.96-8.33$ & .004 & 4.14 & $1.56-10.98$ & - \\
\hline Working Memory & .01 & 3.65 & $1.34-9.89$ & .02 & 3.57 & $1.22-10.48$ & .004 & 4.19 & $1.57-11.20$ & - \\
\hline Plan/Organize & .002 & 5.04 & $1.79-14.20$ & .008 & 4.49 & $1.48-13.65$ & .009 & 3.97 & $1.41-11.21$ & - \\
\hline Org. of Materials & .20 & 2.03 & $0.69-5.99$ & .05 & 3.13 & $1.00-9.82$ & .034 & 3.13 & $1.09-8.94$ & - \\
\hline Monitor & .01 & 4.20 & $1.33-13.28$ & .02 & 4.53 & $1.34-15.34$ & .009 & 4.54 & $1.46-14.15$ & - \\
\hline \multicolumn{11}{|l|}{ BRIEF Self $(\geq 60)$} \\
\hline Inhibit & .006 & 4.10 & $1.50-11.25$ & .04 & 3.37 & $1.09-10.42$ & .07 & 2.64 & $0.92-7.60$ & - \\
\hline Shift & .003 & 3.78 & $1.58-9.03$ & .01 & 3.64 & $1.37-9.68$ & $<.001$ & 6.00 & $2.54-14.19$ & - \\
\hline Emot. Control & .01 & 3.59 & $1.30-9.90$ & .01 & 4.24 & $1.40-12.82$ & .003 & 4.67 & $1.70-12.81$ & - \\
\hline Monitor & .005 & 3.34 & $1.45-7.71$ & .14 & 2.09 & $0.78-5.62$ & .56 & 1.32 & $0.52-3.35$ & $\mathrm{TGA}>\mathrm{SVF}^{* *}(\mathrm{OR}=1.13)$ \\
\hline Working Memory & .04 & 2.09 & $1.03-4.24$ & .15 & 1.85 & $0.80-4.28$ & .006 & $2.69^{\mathrm{a}}$ & $1.33-5.44$ & - \\
\hline Plan/Organize & .006 & $2.92^{\mathrm{a}}$ & $1.36-6.27$ & .08 & 2.24 & $0.91-5.53$ & .10 & 1.98 & $0.88-4.44$ & - \\
\hline Org. of Materials & .11 & 1.76 & $0.88-3.54$ & .32 & 1.54 & $0.66-3.56$ & .42 & 1.36 & $0.65-2.84$ & - \\
\hline Task Completion & .08 & 1.81 & $0.93-3.52$ & .25 & 1.58 & $0.72-3.49$ & .08 & 1.85 & $0.94-3.64$ & - \\
\hline
\end{tabular}

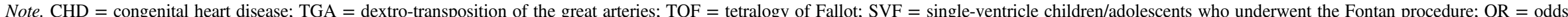

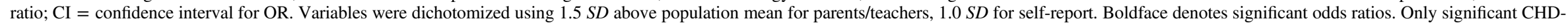
group pairwise comparisons are presented.

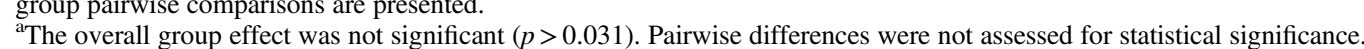

T*p $\leq .01$. 
Table 6. Paired t-test results comparing BRIEF self-report vs. parent/teacher ratings

\begin{tabular}{|c|c|c|c|c|}
\hline & Mean $(95 \% \mathrm{CI})$ & $t$-statistic & $p$-value & Cohen's $d$ \\
\hline & \multicolumn{4}{|c|}{ CHD (combined) } \\
\hline \multicolumn{5}{|c|}{ Self-Report vs. Parent $(n=320)$} \\
\hline Inhibit & $-2.57(-3.80,-1.34)$ & -4.1 & $<.001$ & -0.23 \\
\hline Shift & $-3.49(-4.93,-2.05)$ & -4.76 & $<.001$ & -0.27 \\
\hline Emotion Control & $-2.63(-4.01,-1.25)$ & -3.75 & $<.001$ & -0.21 \\
\hline Monitor & $-5.96(-7.36,-4.56)$ & -8.38 & $<.001$ & -0.47 \\
\hline Working Memory & $-5.71(-7.15,-4.28)$ & -7.83 & $<.001$ & -0.44 \\
\hline Plan/Organize & $-6.22(-7.55,-4.89)$ & -9.22 & $<.001$ & -0.52 \\
\hline Org. of Materials & $-4.76(-5.95,-3.58)$ & -7.89 & $<.001$ & -0.44 \\
\hline \multicolumn{5}{|c|}{ Self-Report vs. Teacher $(n=187)$} \\
\hline Inhibit & $-4.65(-6.55,-2.75)$ & -4.82 & $<.001$ & -0.35 \\
\hline Shift & $-8.03(-10.80,-5.27)$ & -5.73 & $<.001$ & -0.42 \\
\hline Emotion Control & $-4.75(-6.92,-2.59)$ & -4.32 & $<.001$ & -0.32 \\
\hline Monitor & $-8.39(-10.64,-6.14)$ & -7.35 & $<.001$ & -0.54 \\
\hline Working Memory & $-9.83(-12.33,-7.32)$ & -7.74 & $<.001$ & -0.57 \\
\hline Plan/Organize & $-9.77(-12.11,-7.43)$ & -8.25 & $<.001$ & -0.60 \\
\hline \multirow[t]{2}{*}{ Org. of Materials } & $-9.05(-11.70,-6.4)$ & -6.73 & $<.001$ & -0.49 \\
\hline & \multicolumn{4}{|c|}{ Control } \\
\hline \multicolumn{5}{|c|}{ Self-Report vs. Parent $(n=103)$} \\
\hline Inhibit & $-1.28(-3.02,0.46)$ & -1.46 & 0.15 & -0.14 \\
\hline Shift & $-1.40(-3.50,0.70)$ & -1.32 & 0.19 & -0.13 \\
\hline Emotion Control & $-0.38(-2.09,1.34)$ & -0.44 & 0.66 & -0.04 \\
\hline Monitor & $-0.28(-2.50,1.94)$ & -0.25 & 0.80 & -0.02 \\
\hline Working Memory & $1.55(-0.52,3.63)$ & 1.48 & 0.14 & 0.15 \\
\hline Plan/Organize & $-0.42(-2.41,1.57)$ & -0.42 & 0.68 & -0.04 \\
\hline Org. of Materials & $-0.55(-2.69,1.58)$ & -0.51 & 0.61 & -0.05 \\
\hline \multicolumn{5}{|c|}{ Self-Report vs. Teacher $(n=42)$} \\
\hline Inhibit & $-4.31(-7.01,-1.61)$ & -3.22 & 0.002 & -0.50 \\
\hline Shift & $-7.55(-12.41,-2.69)$ & -3.14 & 0.003 & -0.48 \\
\hline Emotion Control & $-6.79(-10.41,-3.16)$ & -3.78 & $<.001$ & -0.58 \\
\hline Monitor & $-5.76(-10.28,-1.24)$ & -2.57 & 0.01 & -0.40 \\
\hline Working Memory & $-2.40(-7.32,2.51)$ & -0.99 & 0.33 & -0.15 \\
\hline Plan/Organize & $-4.81(-8.83,-0.79)$ & -2.42 & 0.02 & -0.37 \\
\hline Org. of Materials & $-0.93(-5.20,3.35)$ & -0.44 & 0.66 & -0.07 \\
\hline
\end{tabular}

to endorse problems with inhibition and self-monitoring. On self-report, the odds of rating oneself as having clinically significant problems with metacognitive skills were statistically no greater among CHD than control participants.

Paired $t$-tests examining differences between BRIEF self-report and parent/teacher ratings showed that, for CHD participants, self-report ratings were lower than parent/teacher scores for all subscales; effect sizes ranged from small to medium (Table 6). Control self-report ratings were significantly lower than teacher ratings for inhibit, shift, emotion control, monitor, and plan/organize subscales, with small to medium effects. Control self- and parent-report ratings were not statistically different.

BRIEF-D-KEFS correlations. Spearman partial correlation coefficients, controlling for SES, age, sex, and race, were calculated between BRIEF and D-KEFS variables for
CHD and control groups separately. Given the large number of variables included, a $p$-value $<.001$ was considered statistically significant. Results are presented in Table 7. For CHD groups, correlations between parent and teacher ratings and several D-KEFS measures (most notably Sorting, Verbal Fluency, and Design Fluency) were statistically significant yet small in magnitude. For controls, BRIEF ratings did not correlate significantly with any D-KEFS variable.

\section{DISCUSSION}

We found that, on average, CHD group means were within the expected age-range on most laboratory EF tasks and informant ratings. However, looking more specifically at areas of clinical impairment, the percentages of children and adolescents performing at least 1.5 SD below the population mean on at least one D-KEFS subtest were nearly twice 
Table 7. Correlations between D-KEFS and BRIEF variables for CHD and control groups

\begin{tabular}{|c|c|c|c|c|c|c|c|c|c|c|c|c|}
\hline & \multicolumn{3}{|c|}{ Verbal Fluency } & \multirow{2}{*}{$\frac{\text { Word Context }}{\text { TCC }}$} & \multicolumn{2}{|c|}{ Sorting } & \multicolumn{3}{|c|}{ Design Fluency } & \multicolumn{3}{|c|}{ Tower } \\
\hline & Letter & Categ & Switch & & CCS & Recog & Filled & Empty & Switch & TAS & TPMR & MAR \\
\hline & \multicolumn{12}{|c|}{ CHD (combined) } \\
\hline \multicolumn{13}{|c|}{ BRIEF Parent $(n=327)$} \\
\hline Inhibit & -0.07 & -0.11 & -0.19 & -0.12 & -0.18 & -0.21 & -0.06 & -0.06 & -0.12 & -0.13 & 0.02 & -0.08 \\
\hline Shift & -0.10 & -0.23 & -0.13 & -0.22 & -0.15 & -0.25 & -0.15 & -0.21 & -0.26 & -0.10 & -0.15 & 0.02 \\
\hline Emotion Control & -0.05 & -0.12 & -0.04 & -0.13 & -0.11 & -0.18 & -0.10 & -0.12 & -0.17 & -0.06 & -0.04 & -0.01 \\
\hline Initiate & -0.07 & -0.13 & -0.18 & -0.12 & -0.13 & -0.21 & -0.13 & -0.13 & -0.19 & -0.15 & -0.14 & -0.05 \\
\hline Working Memory & -0.14 & -0.21 & -0.24 & -0.26 & -0.20 & -0.27 & -0.15 & -0.17 & -0.24 & -0.17 & -0.15 & 0.00 \\
\hline Plan/Organize & -0.11 & -0.19 & -0.19 & -0.14 & -0.16 & -0.22 & -0.13 & -0.12 & -0.19 & -0.17 & -0.10 & -0.04 \\
\hline Org of Materials & 0.04 & 0.01 & -0.08 & 0.00 & 0.03 & -0.03 & 0.01 & 0.02 & 0.01 & -0.05 & 0.05 & -0.06 \\
\hline Monitor & -0.13 & -0.18 & -0.24 & -0.20 & -0.16 & -0.22 & -0.16 & -0.14 & -0.21 & -0.15 & -0.04 & -0.04 \\
\hline \multicolumn{13}{|c|}{ BRIEF Teacher $(n=197)$} \\
\hline Inhibit & -0.05 & -0.01 & -0.14 & -0.19 & -0.15 & -0.13 & -0.09 & -0.13 & -0.07 & -0.12 & -0.02 & -0.01 \\
\hline Shift & -0.19 & -0.11 & -0.17 & -0.30 & -0.28 & -0.27 & -0.19 & -0.23 & -0.20 & -0.10 & -0.13 & 0.05 \\
\hline Emotion Control & -0.05 & -0.05 & -0.14 & -0.17 & -0.13 & -0.11 & -0.07 & -0.12 & -0.13 & -0.12 & -0.04 & 0.02 \\
\hline Initiate & -0.30 & -0.20 & -0.29 & -0.41 & -0.32 & -0.30 & -0.26 & -0.30 & -0.25 & -0.23 & -0.28 & 0.08 \\
\hline Working Memory & -0.21 & -0.14 & -0.22 & -0.39 & -0.27 & -0.26 & -0.18 & -0.26 & -0.20 & -0.19 & -0.18 & 0.07 \\
\hline Plan/Organize & -0.19 & -0.12 & -0.20 & -0.38 & -0.30 & -0.26 & -0.18 & -0.25 & -0.22 & -0.25 & -0.18 & 0.01 \\
\hline Org of Materials & -0.10 & -0.05 & -0.14 & -0.21 & -0.10 & -0.10 & -0.04 & -0.12 & -0.07 & -0.08 & -0.08 & 0.06 \\
\hline Monitor & -0.13 & -0.10 & -0.22 & -0.31 & -0.24 & -0.24 & -0.11 & -0.18 & -0.17 & -0.19 & -0.14 & -0.01 \\
\hline \multicolumn{13}{|l|}{ BRIEF Self $(n=303)$} \\
\hline Inhibit & -0.05 & -0.06 & -0.10 & -0.03 & -0.08 & -0.08 & -0.01 & -0.02 & -0.02 & -0.03 & 0.01 & -0.08 \\
\hline Shift & -0.09 & -0.20 & -0.18 & -0.17 & -0.16 & -0.16 & -0.12 & -0.11 & -0.10 & -0.06 & -0.11 & 0.00 \\
\hline Emotion Control & -0.11 & -0.14 & -0.06 & -0.10 & -0.17 & -0.17 & -0.11 & -0.13 & -0.10 & -0.09 & -0.10 & -0.03 \\
\hline Monitor & -0.03 & -0.15 & -0.13 & -0.09 & -0.11 & -0.13 & -0.06 & -0.08 & -0.03 & -0.11 & -0.05 & -0.11 \\
\hline Working Memory & -0.08 & -0.13 & -0.15 & -0.12 & -0.17 & -0.20 & -0.07 & -0.08 & -0.08 & -0.09 & -0.09 & -0.02 \\
\hline Plan/Organize & -0.09 & -0.17 & -0.13 & -0.09 & -0.14 & -0.13 & -0.04 & -0.04 & -0.07 & -0.07 & -0.06 & -0.03 \\
\hline Org of Materials & -0.03 & -0.08 & -0.05 & 0.08 & 0.02 & 0.02 & 0.05 & 0.05 & 0.01 & 0.01 & 0.01 & -0.03 \\
\hline Task Completion & -0.08 & -0.17 & -0.14 & -0.14 & -0.15 & -0.20 & -0.09 & -0.10 & -0.11 & -0.11 & -0.04 & -0.09 \\
\hline
\end{tabular}


Table 7. (Continued)

\begin{tabular}{|c|c|c|c|c|c|c|c|c|c|c|c|c|}
\hline & \multicolumn{3}{|c|}{ Verbal Fluency } & \multirow{2}{*}{$\frac{\text { Word Context }}{\text { TCC }}$} & \multicolumn{2}{|c|}{ Sorting } & \multicolumn{3}{|c|}{ Design Fluency } & \multicolumn{3}{|c|}{ Tower } \\
\hline & Letter & Categ & Switch & & CCS & Recog & Filled & Empty & Switch & TAS & TPMR & MAR \\
\hline & \multicolumn{12}{|c|}{ Control } \\
\hline \multicolumn{13}{|l|}{ BRIEF Parent $(n=94)$} \\
\hline Inhibit & -0.05 & 0.14 & 0.06 & -0.11 & -0.01 & -0.13 & 0.01 & 0.00 & 0.08 & 0.12 & -0.05 & 0.08 \\
\hline Shift & -0.09 & 0.05 & 0.01 & -0.16 & 0.02 & -0.13 & -0.06 & -0.11 & 0.06 & -0.01 & -0.07 & 0.02 \\
\hline Emotion Control & -0.01 & 0.14 & 0.07 & 0.01 & -0.07 & -0.18 & 0.04 & 0.01 & -0.07 & -0.10 & -0.26 & 0.01 \\
\hline Initiate & -0.02 & -0.06 & -0.05 & -0.06 & -0.03 & -0.28 & -0.03 & -0.14 & 0.05 & 0.04 & -0.12 & -0.01 \\
\hline Working Memory & -0.01 & -0.04 & -0.02 & -0.09 & 0.04 & -0.26 & 0.02 & -0.13 & -0.03 & 0.08 & -0.24 & -0.06 \\
\hline Plan/Organize & -0.14 & 0.03 & -0.04 & -0.02 & 0.00 & -0.23 & -0.06 & -0.10 & 0.00 & 0.01 & -0.18 & -0.13 \\
\hline Org of Materials & 0.03 & 0.12 & 0.10 & 0.12 & 0.01 & -0.09 & -0.16 & -0.16 & 0.00 & 0.10 & -0.02 & -0.11 \\
\hline Monitor & -0.12 & 0.03 & -0.10 & -0.03 & -0.06 & -0.17 & 0.06 & 0.00 & 0.01 & 0.06 & -0.13 & -0.02 \\
\hline \multicolumn{13}{|c|}{ BRIEF Teacher $(n=36)$} \\
\hline Inhibit & -0.12 & 0.20 & -0.13 & -0.15 & -0.18 & -0.50 & -0.15 & -0.04 & -0.23 & 0.05 & -0.31 & 0.14 \\
\hline Shift & 0.04 & 0.19 & 0.02 & 0.10 & 0.09 & -0.33 & 0.18 & 0.02 & -0.28 & 0.18 & -0.38 & 0.35 \\
\hline Emotion Control & 0.02 & 0.17 & 0.09 & 0.23 & -0.09 & -0.27 & -0.11 & -0.19 & -0.29 & -0.01 & -0.32 & 0.06 \\
\hline Initiate & -0.17 & 0.04 & 0.08 & -0.19 & 0.07 & -0.49 & 0.00 & -0.18 & -0.34 & 0.06 & -0.15 & -0.01 \\
\hline Working Memory & -0.23 & 0.05 & -0.13 & -0.10 & -0.13 & -0.40 & -0.33 & -0.33 & -0.30 & -0.10 & -0.33 & -0.05 \\
\hline Plan/Organize & -0.21 & -0.01 & -0.06 & -0.26 & -0.04 & -0.32 & -0.37 & -0.42 & -0.31 & 0.10 & -0.11 & -0.07 \\
\hline Org of Materials & -0.07 & -0.02 & -0.22 & -0.33 & -0.38 & -0.43 & -0.01 & -0.11 & -0.06 & -0.01 & -0.28 & -0.05 \\
\hline Monitor & -0.35 & 0.00 & -0.14 & -0.30 & -0.16 & -0.46 & -0.17 & -0.24 & -0.44 & 0.05 & -0.22 & 0.04 \\
\hline \multicolumn{13}{|l|}{ BRIEF Self $(n=97)$} \\
\hline Inhibit & -0.04 & 0.05 & 0.10 & -0.08 & -0.15 & -0.18 & -0.03 & 0.05 & 0.01 & 0.01 & -0.01 & -0.02 \\
\hline Shift & -0.08 & -0.09 & 0.18 & -0.20 & -0.04 & -0.05 & -0.05 & -0.04 & 0.02 & -0.07 & -0.06 & -0.07 \\
\hline Emotion Control & -0.03 & -0.01 & 0.16 & -0.12 & -0.11 & -0.17 & -0.15 & -0.06 & -0.04 & -0.04 & -0.11 & -0.02 \\
\hline Monitor & -0.15 & -0.06 & 0.09 & -0.19 & -0.02 & -0.09 & -0.05 & -0.08 & 0.05 & 0.05 & -0.06 & 0.01 \\
\hline Working Memory & -0.06 & -0.08 & 0.08 & -0.12 & 0.06 & -0.16 & -0.06 & -0.06 & -0.02 & -0.06 & -0.05 & -0.17 \\
\hline Plan/Organize & -0.14 & -0.18 & 0.01 & -0.17 & -0.05 & -0.16 & -0.05 & -0.07 & 0.07 & -0.15 & -0.08 & -0.12 \\
\hline Org of Materials & 0.01 & -0.06 & 0.03 & -0.07 & 0.10 & -0.16 & -0.15 & -0.09 & 0.07 & -0.06 & -0.03 & -0.14 \\
\hline Task Completion & -0.21 & -0.21 & -0.09 & -0.09 & -0.04 & -0.30 & -0.15 & -0.23 & -0.17 & -0.08 & -0.27 & -0.12 \\
\hline
\end{tabular}

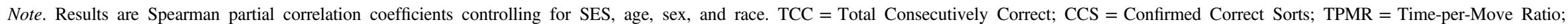
MAR $=$ Move-Accuracy Ratio. Correlations significant at $p<.001$ are bolded. 
as high for CHD groups (75-81\%) than controls (43\%). Percentages of children/adolescents with CHD obtaining clinically elevated scores on the BRIEF were four times higher than controls for parent ratings, and twice as high for teacher and self-report ratings.

Our prediction that controls would outperform CHD groups on all EF measures was partially confirmed: Tower Move-Accuracy Ratio demonstrated very poor sensitivity to differentiate between children with CHD and controls. Controls scored higher than CHD participants on all other D-KEFS measures.

Distinct EF profiles were documented between CHD groups on D-KEFS tasks. Comparing across groups, an interesting pattern emerged in relation to the predominant modality-specific demands of a given task. Controlling for SES, age, sex, and race, CHD was associated with relative deficits in cognitive flexibility/problem-solving and most verbally mediated EF skills for TGA, TOF, and SVF groups. Many visuo-spatially mediated EF skills were also impaired relative to controls in TOF and SVF groups but were relatively preserved in the TGA group.

The etiology of this diagnosis-specific pattern of deficits is unclear but may stem from one or a combination of prenatal, postnatal, and surgical differences. One possibility is that brain regions critical for verbally versus visually mediated $\mathrm{EF}$ abilities may be differentially vulnerable to duration/extent of sub-optimal cerebral perfusion and/or oxygenation in prenatal development. Whereas fetuses with TGA experience reduced oxygenation but relatively normal cerebral perfusion prenatally and immediately after birth, single-ventricle conditions such as HLHS are associated with significant reductions in both oxygenation and perfusion (Licht et al., 2004). Absent in utero antegrade cerebral blood flow, which reduces cerebral perfusion and is common in HLHS (but not TGA), has been linked to reduced volumes of white matter, subcortical gray matter, and regional surface area in fetuses with HLHS (Sethi et al., 2013; Clouchoux et al., 2013) and, as such, may account for relatively greater risk for neural injury and associated functional impairment among single-ventricle participants.

Postnatal cerebral perfusion and oxygenation also differ dramatically across CHD groups. Infants with TGA typically undergo surgical correction within the first weeks of life, essentially normalizing the oxygen content and cerebral perfusion pressure. In contrast, there is much greater variability in age-at-first-surgery for infants with TOF and singleventricle anatomy, with the majority experiencing ongoing alterations in perfusion and oxygenation for months or even years after birth. While children with TOF may undergo repair at a few months old, children with HLHS typically live for 2-3 years with chronic reductions in oxygenation and/or perfusion and continue to experience chronic circulatory changes even after palliation. Surgical management also differs greatly. Most children with TGA require a single postnatal corrective surgery with no additional cardiac operations. Those with TOF may undergo a single correction but typically not until a few months of age. On the other hand, children with single-ventricle conditions nearly always require more than one surgery, which not only exposes them to higher levels of general anesthetics (Jevtovic-Todorovic et al., 2013) and additional opportunities for surgical complications, but also necessitates that they endure a period of chronic hypoxemia while awaiting completion of staged palliation (Fenton, Lessman, Glogowski, Fogg, \& Duncan, 2007).

Finally, although patients were screened for syndromic findings, not all had genetic testing. Indeed, genetic/epigenetic factors influencing patterning of both heart and brain are more common in TOF and single-ventricle conditions than in TGA (Mahle et al., 2013; Newburger et al., 2012; Yi et al., 2014) and as-yet undescribed genetic factors could have contributed to some of the cognitive differences detected. Thus, more severe functional impairment may have been more common in participants with TOF or single-ventricle conditions than in those with TGA for several reasons, ranging from genetic and fetal cerebral hemodynamics to postnatal brain injury.

Regarding informant ratings, parents expressed the widest range of EF concerns, endorsing problems related to both regulatory and metacognitive functions. Teachers also recognized problems with metacognitive skills but in general did not rate children/adolescents with CHD as having more behavior or emotion regulation difficulties than controls. Understanding the nature of this discrepancy likely requires an appreciation of context. Teachers interact with students within the school environment, which is equipped with a range of external regulators (e.g., teachers, non-familial peers, strict scheduling) generally not available within the home that may help to mitigate self-control vulnerabilities. Schools may also present greater demands than home for organization, planning, and independent problem-solving, especially as students transition into middle and high school, which may increase the salience of a child's metacognitive difficulties to his/her teachers. Managing metacognitive challenges at school may also be more effortful for children with CHD, taxing already vulnerable regulatory resources and making it harder for them to effectively modulate their behavior and emotions after school.

It has been suggested that “....relying solely on self-reports of patients with congenital heart disease might underestimate the severity of their challenges, at least in the domain of executive functions" (Bellinger et al., 2014a, p. 9). Consistent with this view, logistic regression analyses were indeed unable to distinguish CHD groups from controls using a cutoff score $\geq 65$. However, application of a more relaxed cutoff score $(\geq 60)$ revealed that children/adolescents with CHD do, in fact, rate themselves as having more problems than controls in select domains of EF. Self-identified concerns regarding cognitive flexibility/shifting, in particular, emerged among members of all three CHD groups, and were consistent with parent ratings and performance on select laboratory flexibility/switching tasks. The development of cognitive flexibility is protracted relative to other core EF abilities (Davidson, Amso, Anderson, \& Diamond, 2006) and is accompanied by increased capacity for switching fluidly 
between rules, accommodating unexpected changes in routine, and generating/entertaining less obvious perspectives than one's own (Diamond, 2013). As such, cognitive flexibility facilitates not only cognitive and academic success but also social competence. Being able to accurately infer the mental states of others (i.e., theory of mind), for example, relies on the ability to toggle flexibly between self- and other-generated representations of the world (Müller, Zelazo, \& Imrisek, 2005) and has been identified as an area of vulnerability among young children with TGA (Calderon et al., 2012). Of interest, individual differences in EF have also recently been shown to predict benefit from theoryof-mind training in healthy preschool children (Benson, Sabbagh, Carlson, \& Zelazo, 2013), suggesting that an understanding of the specific cognitive processes facilitating the development of social cognition in children with CHD may ultimately guide the development of effective prevention/ intervention programs for this vulnerable population as well.

Self- and parent-report ratings also identified concerns regarding emotion regulation. These findings highlight the need for a broader conceptualization of EF in CHD, emphasizing not only decontextualized, "cool" EFs but also the range of "hot" EF skills involved in overcoming problems bearing greater emotional/motivational significance (Prencipe et al., 2011). Mediated by networks involving ventro-medial/ orbitofrontal regions of the prefrontal cortex, as well as the amygdala and limbic structures, "hot" EF skills may be vulnerable to systemic perturbations affecting midline cardiac and neural development. Future studies should harness the power of sensitive behavioral tasks (see Crone \& Van der Molen, 2004; Kerr \& Zelazo, 2004) and neuroimaging techniques to elucidate the developmental course of "hot" EF skills in children with CHD.

Clinically, the self-report ratings collected in this study provide novel insight into how children and adolescents with severe CHD perceive themselves. To date, use of the BRIEF in cardiac neurodevelopmental research has generally been limited to composite variables that collapse across diverse EF skills. This study suggests that children with CHD may be more aware of their struggles than previously thought, particularly regarding problems with cognitive flexibility and emotion regulation. We recommend that clinicians working with the CHD population recognize BRIEF self-report scores $\geq 60$ as potentially "warranting clinical attention" and provide recommendations to manage concerns. We also recommend that future research deconstruct multifaceted composite scores into component subscales to identify specific patterns of risk and protective factors among children with CHD.

This report outlines findings from the largest and most comprehensive study of EF in critical cyanotic CHD to date. There are, however, some limitations to be considered. First, in our effort to limit our sample to children without identified genetic conditions, $25 \%$ of the TOF group was excluded, rendering this group relatively underpowered to detect differences. Second, not all participants had genetic testing or evaluation by a geneticist, leaving open the possibility that some participants with undetected genetic/syndromic conditions may have been included in our sample. Third, it should be acknowledged that the controls in our study were carefully screened for conditions known or expected to adversely impact brain development and thus may be more representative of "super-normal" than "normal" population development (Waber et al., 2007). Our sample was also drawn from studies conducted at a single center and consisted largely of Caucasian participants. Although we attempted to mitigate these issues by controlling statistically for SES and other factors related to EF development, further research is necessary to determine the generalizability of our findings to the broader CHD population. Fourth, because of "task impurity" (Denckla, 1994), performance on tests purported to measure EF can be affected by a range of factors that cannot be adequately accounted for except in tightly controlled experimental paradigms that, unfortunately, were not included in the current study. Fifth, the surgical and postoperative management techniques used in infancy in our mostly adolescent samples might have changed over time in such a way as to produce better outcomes in patients who underwent cardiac surgery more recently. Finally, although the current investigation aimed to operationalize the $\mathrm{EF}$ construct broadly, it will be important for future studies to take this approach further, drawing from developmentally informed models of EF (e.g., Lee, Bull, \& Ho, 2013) to better understand how core EF abilities such as working memory, inhibitory control, and shifting are organized in children with CHD and how these putative core skills may be related to functional outcomes.

In conclusion, the current study demonstrates that children and adolescents developing within the context of critical cyanotic congenital heart disease are at increased risk for $\mathrm{EF}$ deficits. With greater understanding of the specific patterns of cognitive and self-regulatory vulnerabilities experienced by children with $\mathrm{CHD}$, it may be possible to identify risks early and provide individualized supports necessary to promote optimal neurodevelopmental outcomes.

\section{ACKNOWLEDGMENTS}

This research was supported in part by grants from the National Heart, Lung, and Blood Institute (HL77681, HL74734, and HL096825), the Farb Family Fund, and the National Center for Research Resources (RR02172). The authors have no conflicts of interest to declare. The authors thank the children and adolescents, parents, and teachers who participated in this study. The authors also thank Jane Holmes Bernstein, PhD, Caitlin Rollins, MD, and Debbie Waber, PhD, for their thoughtful comments on earlier versions of this manuscript, and Christian Stopp, MS, for his assistance with data management.

\section{REFERENCES}

Bellinger, D.C., Jonas, R.A., Rappaport, L.A., Wypij, D., Wernovsky, G., Kuban, K.C., ... Strand, R.D. (1995). Developmental and neurologic status of children after heart surgery with hypothermic circulatory arrest or low-flow cardiopulmonary bypass. The New 
England Journal of Medicine, 332(9), 549-555. doi:10.1056/ NEJM199503023320901

Bellinger, D.C. (2008). Are children with congenital cardiac malformations at increased risk of deficits in social cognition? Cardiology in the Young, 18(1), 3-9. doi:10.1017/S104795110700176X

Bellinger, D.C., Newburger, J.W., Wypij, D., Kuban, K.C.K., duPlesssis, A.J., \& Rappaport, L.A. (2009). Behaviour at eight years in children with surgically corrected transposition: The Boston Circulatory Arrest Trial. Cardiology in the Young, 19(1), 86-97. doi:10.1017/S1047951108003454

Bellinger, D.C., Rivkin, M.J., DeMaso, D., Robertson, R.L., Stopp, C., Dunbar-Masterson, C., ... Newburger, J.W. (2014a). Adolescents with tetralogy of Fallot: Neuropsychological assessment and structural brain imaging. Cardiology in the Young, 11, 1-10.

Bellinger, D.C., Rivkin, M.J., DeMaso, D., Robertson, R.L., Stopp, C., Dunbar-Masterson, C., \& Wypij, D., Newburger, J.W. (2014b). Neuropsychological and brain structure in adolescents with single ventricle lesions of the heart. (Manuscript in preparation).

Bellinger, D.C., Wypij, D., duPlessis, A.J., Rappaport, L.A., Jonas, R.A., Wernovsky, G., \& Newburger, J.W. (2003). Neurodevelopmental status at eight years in children with dextro-transposition of the great arteries: The Boston Circulatory Arrest Trial. The Journal of Thoracic and Cardiovascular Surgery, 126(5), 1385-1396.

Bellinger, D.C., Wypij, D., Rivkin, M.J., DeMaso, D.R., Robertson, R.L., Dunbar-Masterson, C., ... Newburger, J.W. (2011). Adolescents with d-transposition of the great arteries corrected with the arterial switch procedure: Neuropsychological assessment and structural brain imaging. Circulation, 124(12), 1361-1369. doi:10.1161/ CIRCULATIONAHA.111.026963

Benjamini, Y., \& Hochberg, Y. (1995). Controlling the false discovery rate: A practical and powerful approach to multiple testing. Journal of the Royal Statistical Society. Series B (Methodological), 57(1), 289-300.

Benson, J.E., Sabbagh, M.A., Carlson, S.M., \& Zelazo, P.D. (2013). Individual differences in executive functioning predict preschoolers' improvement from theory-of-mind training. Developmental Psychology, 49(9), 1615-1627.

Brosig, C.L., Mussatto, K.A., Kuhn, E.M., \& Tweddell, J.S. (2007). Neurodevelopmental outcome in preschool survivors of complex congenital heart disease: Implications for clinical practice. Journal of Pediatric Health Care, 21(1), 3-12. doi:10.1016/ j.pedhc.2006.03.008

Calderon, J., Bonnet, D., Courtin, C., Concordet, S., Plumet, M.-H., $\&$ Angeard, N. (2010). Executive function and theory of mind in school-aged children after neonatal corrective cardiac surgery for transposition of the great arteries. Developmental Medicine and Child Neurology, 52(12), 1139-1144. doi:10.1111/j.14698749.2010.03735.x

Calderon, J., Angeard, N., Moutier, S., Plumet, M.-H., Jambaqué, I., \& Bonnet, D. (2012). Impact of prenatal diagnosis on neurocognitive outcomes in children with transposition of the great arteries. The Journal of Pediatrics, 161(1), 94-98.e1. doi:10.1016/ j.jpeds.2011.12.036

Champod, A.S., \& Petrides, M. (2010). Dissociation within the frontoparietal network in verbal working memory: A parametric functional magnetic resonance imaging study. Journal of Neuroscience, 30(10), 3849-3856.

Clouchoux, C., du Plessis, A.J., Bouyssi-Kobar, M., Tworetzky, W., McElhinney, D.B., Brown, D.W., ... Limperopoulos, C. (2013). Delayed cortical development in fetuses with complex congenital heart disease. Cerebral Cortex, 23(12), 2932-2943. doi:10.1093/ cercor/bhs 281
Crone, E.A., \& Van der Molen, M.W. (2004). Developmental changes in real life decision making: Performance on a gambling task previously shown to depend on the ventromedial prefrontal cortex. Developmental Neuropsychology, 25(3), 251-279.

Davidson, M.C., Amso, D., Anderson, L.C., \& Diamond, A. (2006). Development of cognitive control and executive functions from 4-13 years: Evidence from manipulations of memory, inhibition, and task switching. Neuropsychologia, 44, 2037-2078.

Delis, D.C., Kaplan, E., \& Kramer, J.H. (2001). Delis-Kaplan Executive Function System (D-KEFS). San Antonio, TX: The Psychological Corporation.

Denckla, M.B. (1994). Measurement of executive function. In G.R. Lyon (Ed.), Frames of reference for the assessment of learning disabilities: New views on measurement issues (pp. 263-277). Baltimore, MD: Brookes Publishing.

Dennis, M., Francis, D.J., Cirino, P.T., Schachar, R., Barnes, M.A., \& Fletcher, J.M. (2009). Why IQ is not a covariate in cognitive studies of neurodevelopmental disorders. Journal of the International Neuropsychological Society, 15, 331-343.

Diamond, A. (2013). Executive functions. Annual Review of Psychology, 64, 135-168. doi:10.1146/annurev-psych-113011143750

Fenton, K.N., Lessman, K., Glogowski, K., Fogg, S., \& Duncan, K.F. (2007). Cerebral oxygen saturation does not normalize until after stage 2 single ventricle palliation. Annals of Thoracic Surgery, 83(4), 1431-1436.

Gaynor, J.W., Gerdes, M., Nord, A.S., Bernbaum, J., Zackai, E., Wernovsky, G., ... Jarvik, G.P. (2010). Is cardiac diagnosis a predictor of neurodevelopmental outcome after cardiac surgery in infancy? The Journal of Thoracic and Cardiovascular Surgery, 140(6), 1230-1237. doi:10.1016/j.jtcvs.2010.07.069

Gaynor, J.W., Ittenbach, R.F., Gerdes, M., Bernbaum, J., Clancy, R.R., McDonald-McGinn, D.M., ... Spray, T.L. (2014). Neurodevelopmental outcomes in preschool survivors of the Fontan procedure. The Journal of Thoracic and Cardiovascular Surgery, 147, 1276-1283.

Gioia, G., Isquith, P., Guy, S., \& Kenworthy, L. (2000). The Behavior Rating Inventory of Executive Function. Odessa, FL: Psychological Assessment Resources.

Guy, S.C., Isquith, P.K., \& Gioia, G. (2004). Behavior Rating Inventory of Executive Function-Self- Report Version. Odessa, FL: Psychological Assessment Resources.

Hoffman, J.I.E., \& Kaplan, S. (2002). The incidence of congenital heart disease. Journal of the American College of Cardiology, 39(12), 1890-900. Retrieved from http://www.ncbi.nlm.nih.gov/ pubmed/12084585

Hollingshead, A.A. (1975). Four-factor index of social status. Unpublished manuscript. New Haven, CT: Yale University.

Hovels-Gurich, H.H., Konrad, K., Wiesner, M., Minkenberg, R., Herpertz-Dahlmann, B., Messmer, B.J., \& von Bernuth, G. (2002). Long term behavioural outcome after neonatal arterial switch operation for transposition of the great arteries. Archives of Disease in Childhood, 87, 506-510.

Hövels-Gürich, H.H., Konrad, K., Skorzenski, D., Herpertz-Dahlmann, B., Messmer, B.J., \& Seghaye, M.-C. (2007). Attentional dysfunction in children after corrective cardiac surgery in infancy. The Annals of Thoracic Surgery, 83(4), 1425-1430. doi:10.1016/j. athoracsur.2006.10.069

Jevtovic-Todorovic, V., Absalom, A.R., Blomgren, K., Bambrink, A., Crosby, G., Culley, D.J., ‥ Hemmings, H.C. (2013). Anaesthetic neurotoxicity and neuroplasticity: An expert 
group report and statement based on the BJA Salzburg Seminar. British Journal of Anaesthesia, 111(2), 143-151.

Karsdorp, P.A., Everaerd, W., Kindt, M., \& Mulder, B.J.M. (2007). Psychological and cognitive functioning in children and adolescents with congenital heart disease: A meta-analysis. Journal of Pediatric Psychology, 32(5), 527-541. doi:10.1093/jpepsy/js1047

Kerr, A., \& Zelazo, P.D. (2004). Development of "hot" executive function: The Children's Gambling Task. Brain and Cognition, $55,1-10$.

Lee, K., Bull, R., \& Ho, R.M.H. (2013). Developmental changes in executive functioning. Child Development, 84(6), 1933-1953.

Licht, D.J., Wang, J., Silvestre, D.W., Nicolson, S.C., Montenegro, L.M., Wernovsky, G., ... Detre, J.A. (2004). Preoperative cerebral blood flow is diminished in neonates with severe congenital heart defects. The Journal of Thoracic and Cardiovascular Surgery, 128(6), 841-849. doi:10.1016/j.jtcvs.2004.07.022

Licht, D.J., Shera, D.M., Clancy, R.R., Wernovsky, G., Montenegro, L.M., Nicolson, S.C., ... Vossough, A. (2009). Brain maturation is delayed in infants with complex congenital heart defects. The Journal of Thoracic and Cardiovascular Surgery, 137(3), 529-536.

Limperopoulos, C., Tworetzky, W., McElhinney, D.B., Newburger, J.W., Brown, D.W., Robertson, R.L., ... du Plessis, A.J. (2010). Brain volume and metabolism in fetuses with congenital heart disease: Evaluation with quantitative magnetic resonance imaging and spectroscopy. Circulation, 121(1), 26-33. doi:10.1161/ CIRCULATIONAHA.109.865568

Little, D.M., Kraus, M.F., Joseph, J., Geary, E.K., Susmaras, T., Zhou, X.J., \& Gorelick, P.B. (2010). Thalamic integrity underlies executive dysfunction in traumatic brain injury. Neurology, 74(7), $558-564$.

Mahle, W.T., Lu, M., Ohye, R.G., Gaynor, W.J., Goldberg, C.S., Sleeper, LA, ... NewBurger, J.W. (2013). A predictive model for neurodevelopmental outcome after the Norwood procedure. Pediatric Cardiology, 34, 327-333.

Marino, B.S., Lipkin, P.H., Newburger, J.W., Peacock, G., Gerdes, M., Gaynor, J.W., ... Mahle, W.T. (2012). Neurodevelopmental outcomes in children with congenital heart disease: Evaluation and management: A scientific statement from the American Heart Association. Circulation, 126(9), 1143-1172. doi:10.1161/ CIR.0b013e318265ee8a

Miatton, M., De Wolf, D., François, K., Thiery, E., \& Vingerhoets, G. (2007a). Neuropsychological performance in school-aged children with surgically corrected congenital heart disease. The Journal of Pediatrics, 151(1), 73-78, 78.e1. doi:10.1016/j. jpeds.2007.02.020

Miatton, M., De Wolf, D., François, K., Thiery, E., \& Vingerhoets, G. (2007b). Intellectual, neuropsychological, and behavioral functioning in children with tetralogy of Fallot. The Journal of Thoracic and Cardiovascular Surgery, 133(2), 449-455. doi:10.1016/j. jtcvs.2006.10.006

Miller, M., Loya, F., \& Hinshaw, S.P. (2013). Executive functions in girls with and without childhood ADHD: Developmental trajectories and associations with symptom change. Journal of Child Psychology and Psychiatry, 54(9), 1005-1015.

Müller, U., Zelazo, P.D., \& Imrisek, S. (2005). Executive function and children's understanding of false belief: How specific is the relation? Cognitive Development, 20, 173-189.
Newburger, J.W., Jonas, R.A., Wernovsky, G., Wypij, D., Hickey, P.R., Kuban, K.C. K., ... Ware, J.H. (1993). A comparison of the perioperative neurologic effects of hypothermic circulatory arrest versus low-flow cardiopulmonary bypass in infant heart surgery. The New England Journal of Medicine, 329(15), 1057-1064.

Newburger, J.W., Sleeper, L.A., Bellinger, D.C., Goldberg, C.S., Tabbutt, S., Mussatto, K.A., ... Pediatric Heart Network Investigators. (2012). Circulation, 125(17), 2081-2091.

Owen, M., Shevell, M., Majnemer, A., \& Limperopoulos, C. (2011). Abnormal brain structure and function in newborns with complex congenital heart defects before open heart surgery: A review of the evidence. Journal of Child Neurology, 26(6), 743-755. doi:10.1177/0883073811402073

Prencipe, A., Kesek, A., Cohen, J., Lamm, C., Lewis, M.D., \& Zelazo, P.D. (2011). Development of hot and cool executive function during the transition to adolescence. Journal of Experimental Child Psychology, 108(3), 621-637. doi:10.1016/ j.jecp.2010.09.008

Provost, J.S., Petrides, M., \& Monchi, O. (2010). Dissociating the role of the caudate nucleus and dorsolateral prefrontal cortex in the monitoring of events within human working memory. European Journal of Neuroscience, 32(5), 873-880.

Rivkin, M.J., Watson, C.G., Scoppettuolo, L.A, Wypij, D., Vajapeyam, S., Bellinger, D.C., ... Newburger, J.W. (2013). Adolescents with D-transposition of the great arteries repaired in early infancy demonstrate reduced white matter microstructure associated with clinical risk factors. The Journal of Thoracic and Cardiovascular Surgery, 146(3), 543-549.e1. doi:10.1016/j. jtcvs.2012.12.006

Robbins, T.W. (1996). Dissociating executive functions of the prefrontal cortex. Philosophical Transactions of the Royal Society of London, 351, 1463-1471.

Schaefer, C., von Rhein, M., Knirsch, W., Huber, R., Natalucci, G., Caflisch, J., ... Latal, B. (2013). Neurodevelopmental outcome, psychological adjustment, and quality of life in adolescents with congenital heart disease. Developmental Medicine and Child Neurology, 55(12), 1143-1149. doi:10.1111/dmcn.12242

Sethi, V., Tabbutt, S., Dimitropoulos, A., Harris, K.C., Chau, V., Poskitt, K., ... McQuillen, P.S. (2013). Single-ventricle anatomy predicts delayed microstructural brain development. Pediatric Research, 73(5), 661-667.

Stoodley, C.J., \& Schmahmann, J.D. (2010). Evidence for topographic organization in the cerebellum of motor control versus cognitive and affective processing. Cortex, 46(7), 831-844.

Waber, D.P., De Moor, C., Forbes, P.W., Almli, C.R., Botteron, K.N., Leonard, G., ...The Brain Development Cooperative Group. (2007). Journal of the International Neuropsychological Society, 13, 729-726.

Watanabe, K., Matsui, M., Matsuzawa, J., Tanaka, C., Noguchi, K., Yoshimura, N., ... Gur, R.C. (2009). Impaired neuroanatomic development in infants with congenital heart disease. The Journal of Thoracic and Cardiovascular Surgery, 137(1), 146-153. doi:10.1016/j.jtcvs.2008.06.036

Yi, J.J., Tang, S.X., McDonald-McGinn, D.M., Calkins, M.E., Whinna, D.A., Souders, M.C., ... Gur, R.E. (2014). Contribution of congenital heart disease to neuropsychiatric outcome in schoolage children with 22q11.2 deletion syndrome. American Journal of Medical Genetics Part B Neuropsychiatric Genetics, 165, 137-147. 